\title{
Older Adults with Intellectual Disability
}

Authors: Amanda Sinai ${ }^{1}$, Ingrid Bohnen ${ }^{2}$, Andre Strydom ${ }^{1,3}$

1. Mental Health Sciences Unit, University College London, UK

2. Westminster Learning Disability Partnership, London, UK

3. Islington Learning Disabilities Partnership, London, UK

Corresponding author: Andre Strydom, UCL Mental Health Sciences Unit Charles Bell House, 2nd Floor, 67-73 Riding House Street, London W1W 7EJ. a.strydom@ucl.ac.uk, tel: 0207679 9308.

Conflicts of Interest and Source of Funding Acknowledgements: Andre Strydom is an investigator and Amanda Sinai is a rater in a trial sponsored by Roche to improve cognition in adults with Down syndrome. Andre Strydom and Ingrid Bohnen work for the National Health Service (NHS). Amanda Sinai is a Research Associate, funded by the Baily Thomas Charitable Fund. 


\title{
Older Adults with Intellectual Disability
}

\begin{abstract}
Purpose of review: Improvements in health and social care for people with intellectual disability have led to a dramatic increase in the life expectancy of this population, resulting in a large and growing number of older adults with intellectual disability. They are at risk of age-related mental disorders such as dementia, and continue to present with high rates of mental illness.
\end{abstract}

Recent findings: Recent research with older adults with intellectual disability has included physical health issues that may affect mental well-being, and biological and clinical features of dementia in people with Down syndrome. Two key clinical trials, of memantine and antioxidants for dementia in Down syndrome showed that these treatment options were ineffective in the short term. Other research focused on environmental and psychosocial issues (including carer issues) and end of life care.

Summary: Improved mental well-being requires effective management of mental illness and co-morbid physical health problems as well as consideration of environmental and social issues. Biological research has identified potential treatments for age-related decline which has lead to development of medication trials. Despite disappointing results, two recent medication trials showed that randomised controlled trials are feasible in older people with intellectual disabilities - a group who are often excluded from trials.

Keywords: Ageing, Dementia, Intellectual disability, Older adults.

\section{Introduction}

Improvements in health and social care for people with intellectual disability have led to a dramatic increase in the life expectancy of this population, which is resulting in a large and growing number of older adults with intellectual disability. This has been particularly striking in the Down syndrome population - the population of people with Down syndrome over 40 years old has doubled in Northern European countries since 1990 [1**], while the mean life expectancy of people with intellectual disability of any cause in the 1930s was estimated at 18.5 years, which increased to 66 years in the 1990s [2].

Older adults are at risk of age-related disorders, and people with intellectual disability may be particularly challenged by declines in health, symptoms such as pain [3] and increased dependency. Cognitive decline further reduces a person's ability to cope and adapt. 
This review will consider recent research (August 2010 - March 2012) on mental disorders in people with intellectual disability, including biological, health and psychosocial factors, its impact on carers and care systems, and advances in treatment options.

\section{Epidemiology of mental disorders in older adults with intellectual disability}

The prevalence of many mental health problems remains relatively stable as people age [4], regardless of whether they have co-morbid autism [5]. However, prevalence of depression was found to be significantly higher among older adults with intellectual and other developmental disabilities (aged 55 and over) in Canada compared to matched peers from the general population [6], while problem behaviours tend to decrease with age [4]. Mental illness adds significantly to carer burden [7] and has a significant independent relationship with cost of care in older adults with intellectual disability [8].

Rates of dementia in the population with intellectual disability not due to Down syndrome are comparable to or higher than the general population, while Alzheimer's disease in people with Down syndrome increases from under $10 \%$ in their 40 s to more than $30 \%$ in their 50s, with some studies reporting prevalence rates in excess of 50\% for those 60 and older (see Strydom et al 2010 [9] for a review). Dementia may be diagnosed four times more often in older people with intellectual and other developmental disabilities than in the general population [6].

\section{Physical health in older adults with intellectual disability}

Physical health problems are often linked with poorer mental health. There have been several recent studies of the physical health issues of older people with an intellectual disability. These studies indicate that functional restrictions, multi-morbidity, an unhealthy lifestyle, limited detection of risk factors for cardiovascular disease, limited participation in screening programmes and missed diagnoses due to a lack of subjective complaints are all significant factors in this population [10].

A Dutch cross-sectional study of people with intellectual disability aged 50 and over living in a variety of settings showed a higher prevalence of obesity than in the general older Dutch population. Use of atypical antipsychotic medication was significantly associated with being overweight or obese, along with physical inactivity, being female, having Down syndrome, being older, having less severe intellectual disability, autism, and higher independency in terms of preparing meals $\left[11^{*}\right]$.

People with Down syndrome experience premature ageing including cataracts [12] and presbycusis [13], which could impact on their function and mental state. The pattern of physical health issues associated with Down syndrome changes as they age - a recent 
Finnish population based study of case records of people with Down's syndrome [14] found that congenital heart defects and middle ear infections were mostly experienced by younger people, while thyroid disease, epilepsy, and Alzheimer's disease were frequent among older people. Women with Down syndrome often experience early menopause, which has been associated with an increased risk of dementia and decreased life expectation [15**].

The European POMONA II study [16**] focussed on age-specific differences relating to environment and lifestyle factors and on the prevalence of specific medical conditions in people with intellectual disability. It showed that rates of smoking and use of alcohol were lower than in the general population but were higher with older age. Over $60 \%$ of older adults had a sedentary lifestyle. Cataract, hearing disorder, diabetes, hypertension, osteoarthritis/arthrosis, and osteoporosis were positively associated with increasing age, while allergies and epilepsy were negatively associated. Furthermore, some evidence of health disparities was found for older people with intellectual disability, particularly in terms of underdiagnosed or inadequately managed preventable health conditions.

\section{Dementia}

Recent publications from the USA [17] and Britain [18] provide important guidelines on dementia in people with intellectual disabilities. Diagnosis of dementia is often difficult in people with pre-existing cognitive deficits, and it is therefore important to establish the validity of diagnostic criteria in older adults with intellectual disability. ICD10 and DSM IV dementia criteria both showed substantial reliability and satisfactory validity, although caution is advised when using these criteria in people with more severe intellectual disability and/or co-morbid sensory disabilities [19*].

Most of the recent research in dementia in people with intellectual disability has focused on Down syndrome and it is hoped that by understanding of the pathophysiology of dementia in this population, it will also further the understanding of dementia in the general population.

Some recent research on clinical features of dementia in people with Down syndrome has focussed on pre-clinical dementia or the early stages of dementia in this population. In a longitudinal study of 30 adults with Down syndrome, individuals with cognitive deterioration showed significant changes in behaviour and decreases in executive function measures when compared to the group with no cognitive deterioration during the study period [20]. The authors highlight that specific behavioural changes can be seen even in the early stages of cognitive deterioration, which is in keeping with the findings of another study of adults with Down syndrome without a diagnosis of Alzheimer's disease ( $n=78)$, which identified early onset of disinhibited behaviours in a large proportion [21]. The authors hypothesised that the high rates of disinhibition in the preclinical stages of dementia in 
people with Down syndrome may reflect a disproportionately affected orbito-frontal circuit, which is mediated by serotonin. Further research would be important to confirm this.

The amyloid cascade hypothesis remains the most prominent theory for the pathophysiology of Alzheimer's disease and is of much interest in Down syndrome, as the APP gene on chromosome 21 is strongly implicated in the development of Alzheimer's neuropathology in these patients. Recent advances in Positron Emission Tomography (PET) imaging using new ligands have enabled researchers to show cerebral amyloid and tau in people with Down syndrome. In a proof-of-principle study, a marker of cerebral amyloid- $\beta$ (11-labelled Pittsburgh Compound $\mathrm{B},\left[{ }^{11} \mathrm{C}\right] \mathrm{PiB}$ ) was found to have significant binding in people with Down syndrome who were older than 45 years, whether or not they had clinical features of dementia $\left[22^{* *}\right]$ and shows that $\left[{ }^{11} \mathrm{C}\right]$ PiB PET can be successfully used in this population. In another PET study, $\left[{ }^{18} \mathrm{~F}\right]$ FDDNP (a ligand that detects both amyloid and tau) has been shown to have high binding levels in adults with Down syndrome, which (in some brain regions) was correlated to age and behavioural dysfunction and comparable to findings in participants with Alzheimer's disease from the general population [23**].

The relationship between amyloid- $\beta$ levels in plasma and cognitive functioning in adults with Down syndrome has also been explored - plasma amyloid- $\beta$ levels did not dissociate between adults with Down syndrome with and without dementia, but ApoE4 was associated with higher levels of amyloid- $\beta 40$ but not amyloid- $\beta 42$ in those with dementia [24]. Although biomarkers are being considered for the diagnosis of Alzheimer's disease [25], the clinical application of plasma amyloid- $\beta$ and amyloid or tau PET scans is yet to be established.

Improved understanding of the neurobiology of Down syndrome is now leading to potential treatment options for improving cognitive functioning or modifying the dementia process in people with Down syndrome. For example, Down syndrome mouse model studies showed that NMDA receptor signalling is defective in Down syndrome, which is associated with impaired hippocampal function. Treatment of Down syndrome mice with memantine (a $\mathrm{N}$ methyl-D-aspartate (NMDA) receptor antagonist that improves cognitive function in older adults with moderate-to-severe Alzheimer's disease in the general population) resulted in improved learning and memory (see Costa for a review [26]). This observation has led to several trials of memantine in Down syndrome.

The MEADOWS trial was a 52-week double blind, randomised, placebo controlled trial of memantine in 173 people with Down syndrome with and without dementia [27**]. Both groups showed reductions in function and cognition over the course of the study, but there were no differences between the groups for any of the outcomes.

Oxidative stress is also thought to play a role in the pathophysiology of Alzheimer's disease and is particularly of interest in Down syndrome, as the dosage sensitive superoxide dismutase gene (SOD1) is located on chromosome 21 . Oxidative stress in people with Down 
syndrome and the potential for antioxidant supplements is explored further in two recent review articles $[28,29]$. A two year randomised controlled trial of antioxidant supplementation (900IU alpha-tocopherol, $200 \mathrm{mg}$ ascorbic acid and $600 \mathrm{mg}$ alpha-lipoic acid) in people with Down syndrome and dementia was conducted by Lott et al [30**]. Their findings were similar to studies of antioxidants in people with Alzheimer's disease in the general population, as participants receiving antioxidant supplements did not show an improvement in cognitive function or stabilisation of cognitive deterioration when compared to the placebo group.

\section{Social and environmental issues}

Appropriate social care and support and a comfortable and suitable living environment is conducive to good mental health. This link between social and environmental issues and mental health is highlighted in an important recent study from the Netherlands, which studied the association between life events and symptoms of anxiety and depression in older adults with intellectual disability [31**]. Depression and anxiety were assessed using self-report and informant-report screening instruments $(n=988)$ and psychiatric interviews ( $n=286$ ), and $72 \%$ of participants had been exposed to one or more negative life events in the preceding year. Minor physical illness or injury and problems with fellow residents were found to be significantly associated with both increased depressive and anxiety symptoms. Decline or loss of mobility and loss of leisure-time activities were both found to be significantly associated with depressive symptoms, but causality cannot be assumed, as the study design is cross-sectional.

The benefits of daytime activities and community based services for older adults with disabilities, including those with intellectual disabilities, presents as a common theme in two qualitative studies based in Scotland [32] and Australia [33] respectively. In Norway, where senior centres are visited by approximately $40-50 \%$ of the population aged 67 years or older, participants without intellectual disabilities who attended these centres considered the potential barriers and solutions to inclusion of people with intellectual disability [34]. Barriers included attitude towards intellectual disability and personal, organisational and economic issues. Suggested solutions included financing the centres so that appropriate support could be provided for individuals with intellectual disability.

Several studies have considered the issues of access to support and housing associated with ageing with an intellectual disability. One qualitative study from Australia conducted focus groups and individual interviews with 15 people with intellectual disability and 10 family carers. Themes that emerged included the issues associated with transition from informal to formal housing and support services. The majority of family members interviewed were concerned that the residential aged care sector would struggle to meet the needs of older people with intellectual disabilities, who were often younger than the usual resident [35], 
while a cross-sectional study in Taiwan found that more than half of managers of institutions that care for people with intellectual disabilities were not satisfied with their provisional care of ageing residents with intellectual disability [36].

Specific issues related to supporting people with Down syndrome and advanced dementia are highlighted in a qualitative study which involved focus groups of staff working in six intellectual disabilities services and a palliative care service in Ireland. Readiness to respond to end of life needs, fear of swallowing difficulties and environmental concerns and ageing in place were common themes [37].

A proposed quality measurement framework in group home dementia care for people with intellectual disabilities [38] included: 1 . Clinically relevant and early periodic assessment, 2. Functional modifications in the long-term setting, 3 . Constructive staff education and functionality for stage-adapted care, and 4 . Flexible long term service provision that recognises and plans for the progression of decline and loss of function.

\section{Carer issues}

Family carers play an important - frequently the most important - role in supporting people with intellectual disability. As individuals with intellectual disability grow older, so do their family carers. This may bring with it a change in the needs of the carer and a change in their abilities to care for their loved ones (in particular, due to physical limitations).

Two recent studies have examined factors that may be important in older carers of adults with intellectual disability. One large study, based in Taiwan, interviewed 350 female carers aged 55 years and over. Rates of self-reported depressive symptoms in these carers were high, and were significantly associated with self-reported carer physical health, caregiver burden and lack of social support [39*].

An Australian study found the caring role may be rewarding as well as stressful - highlighting the importance of recognising both the benefits and burdens of caring [40].

\section{End of life issues}

Age related medical conditions such as late onset seizures, pneumonia, fractures and other emerging medical problems - many requiring hospitalisation - have been found to be significant events for adults with Down syndrome which can contribute to their decline in health status and adaptive functioning [41].

Ageing adults with Down syndrome experienced more relocations and other life events than adults with intellectual disabilities aged 50 and older without Down syndrome [42]. Age related functional decline and the higher incidence of dementia were implicated as the 
contributing factors that lead to relocation and nursing home placement. Mortality was significantly earlier in the Down syndrome group with the mean age at death to be 61.4 years compared with 73.2 years in those without Down syndrome.

The end of life care and treatment of people with an intellectual disability is likely to vary. End-of-life care policies in Flemish residential care facilities were found to be often absent and their content was not very specific for people with intellectual disability [43]. Although some had a policy on palliative care, it appeared that end-of-life decisions with a possible life-shortening effect were rarely mentioned and elaborated on.

People with intellectual disability may have only partial understanding of the concept of death, leaving them vulnerable to misinterpretations. There is scope for making sense of death by using religious and spiritual themes and by providing teaching on the biological explanations of the life cycle [44].

\section{Conclusion}

Recent research with older adults with intellectual disability has focused on a number of different areas, including physical health issues, biological and clinical features of dementia in people with Down syndrome and psychosocial issues (including carer issues). Potential treatments for cognitive decline have been identified using basic sciences research, for example using Down syndrome mouse models, which is now being translated into humans and has lead to development of medication trials in people with Down syndrome. The recent memantine and antioxidant trails in older adults with Down syndrome highlight that randomised controlled trials are feasible in older people with intellectual disabilities - a group who are often excluded from clinical trials.

Future research should build on our increasing understanding of the neurobiology of intellectual disabilities and ageing associated mental disorders, while pursuing potential treatment options in clinical trials. Effective care environments and interventions to reduce carer burden are equally important topics, as is exploring ways to improve end-of-life care.

\section{Key Points}

- Mental health issues and dementia are common in older adults with intellectual disability

- Recent research has included physical health issues that may affect mental wellbeing, biological and clinical features of dementia in people with Down syndrome and psychosocial issues, including carer issues. 
- The recent memantine and antioxidant trails in older adults with Down syndrome highlight that randomised controlled trials are feasible in older people with intellectual disabilities.

\section{References}

**1. de Graaf G, Vis JC, Haveman M, et al. Assessment of Prevalence of Persons with Down Syndrome: A Theory-based Demographic Model. Journal of Applied Research in Intellectual Disabilities 2011; 24:247-262.

This paper used existing epidemiological data from several Northern European countries and modelled the current and future population prevalence of Down syndrome.

2. Braddock D. Ageing and developmental disabilities: Demographic and policy issues affecting American families. Mental Retardation 1999; 37:155-161.

3. Walsh M, Morrison TG, McGuire BE. Chronic pain in adults with an intellectual disability: Prevalence, impact, and health service use based on caregiver report. Pain 2011; 152:19511957.

4. Hove O, Havik OE. Developmental level and other factors associated with symptoms of mental disorders and problem behaviour in adults with intellectual disabilities living in the community. Soc Psychiatry Psychiatr Epidemiol 2010; 45:105-13.

5. Totsika V, Felce D, Kerr M, Hastings RP. Behavior Problems, Psychiatric Symptoms, and Quality of Life for Older Adults with Intellectual Disability with and without Autism. Journal of Autism and Developmental Disorders 2010; 40:1171-1178.

6. Shooshtari S, Martens PJ, Burchill CA,et al. Prevalence of Depression and Dementia among Adults with Developmental Disabilities in Manitoba, Canada. Int J Family Med 2011; Article ID:319574. Epub 2011 Aug 8. doi:10.1155/2011/319574.

7. Courtenay K, Jokinen NS, Strydom A. Caregiving and Adults with Intellectual Disabilities Affected by Dementia. Journal of Policy and Practice in Intellectual Disabilities 2010; 7: 2633.

8. Strydom A, Romeo R, Perez-Achiaga N, et al. Service use and cost of mental disorder in older adults with intellectual disability. British Journal of Psychiatry 2010; 196:133-138.

9. Strydom A, Shooshtari S, Lee L, et al. Dementia in Older Adults With Intellectual Disabilities-Epidemiology, Presentation, and Diagnosis. Journal of Policy and Practice in Intellectual Disabilities 2010; 7:96-110.

10. Evenhuis HM. Health of the mentally handicapped elderly/Gezondheid van ouderen met verstandelijke handicaps. Nederlands Tijdschrift voor Geneeskunde 2011; 155:A2598. 
*11. de Winter CF, Bastiaanse LP, Hilgenkamp TIM, et al. Overweight and obesity in older people with intellectual disability. J Intellect Dev Disabil 2011; 36:118-26.

This study highlights the higher prevalence of obesity in older adults with intellectual disability.

12. Van Splunder J, Stilma JS, Bernsen RMD, Evenhuis HM. Prevalence of ocular diagnoses found on screening 1,539 adults with intellectual disabilities. Ophthalmology 2004; 111: 1457-1463.

13. Meuwese-Jongejeugd A, Vink M, van Zanten $B$, et al. Prevalence of hearing impairment in adults with an intellectual disability: cross sectional population study. Int J Audiol 2006; 45:660-669.

14. Määttä T, Määttä J, Tervo-Määttä T, et al. Healthcare and guidelines: a population-based survey of recorded medical problems and health surveillance for people with Down syndrome. Journal of Intellectual and Developmental Disability 2011; 36:118-126.

**15. Coppus AMW, Evenhuis HM, Verbene GJ, et al. Early age at menopause is associated with increased risk of dementia and mortality in women with Down syndrome. J Alzheimers Dis 2010; 19:45-50.

This study highlights the link between early age at menopause and dementia in women with Down syndrome.

**16. Haveman M, Perry J, Salvador-Carulla L, et al. Ageing and health status in adults with intellectual disabilities: Results of the European POMONA II study. Journal of Intellectual \& Developmental Disability 2011; 36: 49-60.

This is a large, cross-sectional study which looked at health in older adults with intellectual disability living in 14 European member states. It provides detailed information about health conditions and health disparities in this population.

17. National Task Group on Intellectual Disabilities and Dementia Practice. "My Thinker's not Working": A National Strategy for Enabling Adults with Intellectual Disabilities Affected by Dementia 2012; National Task Group on Intellectual Disabilities and Dementia Practice.

18. British Psychological Society, Royal College of Psychiatrists. Dementia and people with learning disabilities. Leicester: British Psychological Society; 2009.

*19. Strydom A, Chan T, Fenton C. Validity of Criteria for Dementia in Older People With Intellectual Disability. American Journal of Geriatric Psychiatry 2012; doi: 10.1097/JGP.0b013e31824472be (epub ahead of print)

This study found that ICD10 and DSM IV dementia criteria showed substantial reliability and satisfactory validity when used in people with intellectual disability. 
20. Adams $D$, Oliver $C$. The relationship between acquired impairments of executive function and behaviour change in adults with Down syndrome. Journal of Intellectual Disability Research 2010; 54; 393-405.

21. Ball SL, Holland AJ, Watson PC, Huppert FA. Theoretical explanation of the neural bases of behavioural disinhibition, apathy and executive dysfunction in preclinical Alzheimer's disease in people with Down's syndrome: potential involvement of multiple frontalsubcortical neuronal circuits. Journal of Intellectual Disability Research 2010; 54:320-336.

**22. Landt J, D'Abrera C, Holland AJ et al. Using Positron Emission Tomography and Carbon 11-Labeled Pittsburgh Compound B to Image Brain Fibrillar $\beta$-Amyloid in Adults with Down Syndrome. Arch Neurol 2011; 68:890-896.

This proof-of-principle study found that a marker of cerebral amyloid- $\beta$ (11-labelled Pittsburgh Compound $\left.\mathrm{B},{ }^{11} \mathrm{C}\right] \mathrm{PiB}$ ) had significant binding in people with Down syndrome who were older than 45 years. It shows that $\left[{ }^{11} \mathrm{C}\right]$ PiB PET can be successfully used in this population.

**23. Nelson LD, Siddarth P, Kepe $V$ et al. Positron Emission Tomography of Brain $\beta-$ Amyloid and Tau levels in Adults with Down Syndrome. Arch Neurol 2011; 68:768-774.

This study shows that $\left[{ }^{18} \mathrm{~F}\right]$ FDDNP (a ligand that detects both amyloid and tau) has high binding levels in adults with Down syndrome, which (in some brain regions) was correlated to age and behavioural dysfunction and comparable to findings in participants with Alzheimer's disease from the general population.

24. Head E, Doran E, Nistor $M$ et al. Plasma Amyloid- $\beta$ as a Function of Age, Level of Intellectual Disability, and Presence of Dementia in Down Syndrome. Journal of Alzheimer's Disease 2011; 23:399-409.

25. Dubois $B$, Feldman $\mathrm{HH}$, Jacova $\mathrm{C}$ et al. Revising the definition of Alzheimer's disease: $a$ new lexicon. Lancet Neurology 2010; 9:1118-1127.

26. Costa ACS. On the Promise of Pharmacotherapies Targeted at Cognitive and Neurodegenerative Components of Down Syndrome. Developmental Neuroscience 2011; 33:414-427.

**27. Hanney $\mathrm{M}$, Prasher $\mathrm{V}$, Williams $\mathrm{N}$ et al. Memantine for dementia in adults older than 40 years with Down's syndrome (MEADOWS): a randomised, double-blind, placebo controlled trial. Lancet 2012; 379:528-536.

This was a double blind, randomised, placebo controlled trial of memantine in people with Down syndrome with and without dementia. It highlights that randomised controlled trials are feasible and important in older people with intellectual disabilities. 
28. Perluigi M, Butterfield DA. Oxidative Stress and Down Syndrome: A Route toward Alzheimer-Like Dementia. Current Gerontology and Geriatrics Research 2012; Article ID 724904,10 pages. doi:10.1155/2012/724904

29. Lott IT. Antioxidants in Down syndrome. Biochemica et Biophysica Acta 2012; 1822: 657663.

**30. Lott IT, Doran E, Nguyen VQ et al. Down Syndrome and Dementia: A Randomized, Controlled Trial of Antioxidant Supplementation. American Journal of Medical Genetics Part A 2011; 155A:1939-1948.

This was a two year randomised controlled trial of antioxidant supplementation in people with Down syndrome and dementia. It highlights that randomised controlled trials are feasible and important in older people with intellectual disabilities.

**31. Hermans H, Evenhuis HM. Life events and their associations with depression and anxiety in older people with intellectual disabilities: Results of the HA-ID study. Journal of Affective Disorders 2012; 138;79-85.

This cross-sectional study highlights the association between some life events and symptoms of anxiety and depression in older adults with intellectual disability.

32. Judge J, Walley R, Anderson B, Young R. Activity, Ageing and Retirement: The Views of a Group of Scottish People with Intellectual Disabilities. Journal of Policy and Practice in Intellectual Disabilities 2010; 7:295-301.

33. Ellison C, White A, Chapman L. Avoiding institutional outcomes for older adults living with disability: The use of community-based aged care supports. Journal of Intellectual and Developmental Disability 2011; 36:175-183.

34. Ingvaldsen AK, Balandin S. "If We are Going to Include Them We have to do it Before We Die": Norwegian Seniors' Views of Including Seniors with Intellectual Disability in Senior Centres. Journal of Applied Research in Intellectual Disabilities 2011; 24:583-593.

35. Shaw K, Cartwright C, Craig J. The housing and support needs of people with an intellectual disability into older age. Journal of Intellectual Disability Research 2011; 55:895903.

36. Lin J-D, Wu C-L, Lin P-Y, et al. Early onset ageing and service preparation in people with intellectual disabilities: Institutional managers' perspective. Research in Developmental Disabilities 2011; 32:188-193.

37. McCarron M, McCallion P, Fahey-McCarthy E, et al. Supporting persons with Down syndrome and advanced dementia. Dementia 2010; 9:285-298. 
38. Janicki MP. Quality outcomes in group home dementia care for adults with intellectual disabilities. Journal of Intellectual Disability Research 2011; 55:763-776.

*39. Chou YC, Pu C-Y, Fu L-Y, Troger T. Depressive symptoms in older female carers of adults with intellectual disabilities. Journal of Intellectual Disability Research 2010; 54:1031-1044.

This cross-sectional study found that rates of self-reported depressive symptoms in older carers of people with intellectual disability were high.

40. Llewwllyn G, McConnell D, Gething L, et al. Health status and coping strategies among older parent-carers of adults with intellectual disabilities in an Australian sample. Research in Developmental Disabilities 2010; 31:1176-1186.

41. Patti PJ, Amble K, Flory M. Life events in older adults with intellectual disabilities: differences between adults with and without Down syndrome. Journal of Policy and Practice in Intellectual Disabilities 2005; 2:149-55.

42. Patti PJ, Amble K, Flory. Placement, relocation and end of life issues in aging adults with and without Down's syndrome: a retrospective study. Journal of Intellectual Disability Research 2010; 54:538-546.

43. D'Haene I, Pasman HRW, Deliens L, et al. End-of-life care policies in Flemish residential care facilities accommodating persons with intellectual disabilities. Journal of Intellectual Disability Research 2010; 54:1067-1077.

44. McEvoy J, MacHale R, Tierney E. Concept of death and perceptions of bereavement in adults with intellectual disabilities. Journal of Intellectual Disability Research 2012; 56;191203. 